\title{
ANALYSIS OF FACTORS AFFECTING THE BENEFITS OF DEMAND INFORMATION SHARING
}

\author{
Hyun-Woong Jin
}

\section{Introduction}

Globalization increases the complexity of supply chain and companies are faced with competitive pressure and environmental uncertainties as well as increasing customer demand (Thomas \& Esper, 2010). To satisfy the fluctuating customer demand and to make various players in a supply chain align with customer requirement, collaboration between the players based on the timely communication is required. Moreover, sharing important information such as customer demand and on-hand inventory level with other players is required to coordinate the players' activities.

With respect to the inventory management, various collaborative policies such as $Q R$ (Quick Response), ECR (Efficient Customer Response), VMI (Vendor Managed Inventory) and CPFR (Collaborative Planning, Forecasting and Replenishment) have been developed so as to overcome the limitation of standalone inventory management policies. These collaborative inventory management policies are based on the sharing of customer demand information between players in the supply chain. Sharing customer demand information across the supply chain is as an effective approach to improve the performance of the whole supply chain by reducing demand distortion which is the main source of the bullwhip effect.

Even though the sharing of customer demand information provides many benefits such as reducing the inventory level and improving the customer service level, the amount of benefits depends heavily on the business environment where the supply chain belongs. And demand information sharing between companies requires a large amount of budget to install infra-structure for the real time information access. (Yigitbasioglu, 2010; Costantino et al., 2015). In addition, demand information sharing leads to a change in the work process within the organization and it might be faced with the employee resistance. Therefore, it is necessary for the management teams to verify whether the sharing of customer demand information is beneficial to them by considering their business environment and what is needed to improve the benefit of demand information sharing.

As a first step into the collaboration with other players, demand information sharing has been an interesting research topic to the SCM researchers. Among them, the effect of demand information sharing on the supply chain performance has been one of the main issues, because these researches may provide a clue as to whether some companies can enjoy the benefit of collaborative inventory management policy or not. Therefore, in this paper, the benefit of demand information sharing under various business environments is studied in order to provide some managerial implications for the companies considering the adoption of a collaborative inventory management policy with external companies.

The rest of this paper is organized as follows: Section 1 reviews the literature related to this research. Section 2 describes the system dynamics model developed in this paper and Section 3 discusses the results of numerical experiments. Section 4 makes concluding remarks.

\section{Literature Review}

As mentioned above, various researches dealing with the benefit of demand information sharing have been published. For example, Lee et al. (1997) mentioned that the distortion of customer demand is one of the main sources of the bullwhip effect and claimed that sharing the information on customer demand could reduce the on-hand inventory level of each player in the supply chain. Yu et al. (2001) performed the case study on a mobile phone supply chain 
and found that information sharing brought the reduction of inventory cost as well as the inventory level itself.

The above researches utilized the qualitative methodology to derive their managerial insights. As well as the qualitative methodology, various analytical approaches have also been proposed to measure the effect of information sharing. Chen et al. (2000) considered a two-echelon supply chain and constructed a mathematical model to measure the bullwhip effect of the supply chain. By analyzing the mathematical model, they showed that the supply chain with complete access to the customer demand information could significantly reduce the bullwhip effect. Sabitha et al. (2016) considered two different scenarios, supply chain wide information sharing and $\mathrm{VMI}$, and quantified their benefits based on the constructed mathematical models.

The advantage of analytical approach is to provide a clear conclusion within the considered specific system. However, it is not easy to apply its results to different systems since the results are based on many assumptions and a limited environment. In this point, another useful approach to analyze the benefit of demand information sharing is the simulation study. Even though the results derived from the simulation study do not guarantee the optimality, these can provide some meaningful insights on the behavior of the considered system under various environments. Therefore, the simulation study helps decision makers by providing the expected results of the considered system with much less cost than the field experiment which requires large investment and even is not feasible (Zhang \& Zhang, 2007).

For example, Lee et al. (2000) compared the benefit of information sharing under some business environments. In their research, the distribution and parameters of customer demand were shared with manufacturer by retailer. The authors monitored the differences in the benefit of demand information sharing when the variance of demand, the correlation of demand and the lead time were changed. Based on the simulation results, they concluded that the benefit of demand information sharing might increase when the variance of demand was high, daily demands were highly correlated over time, and the lead time was long.

Zhang and Zhang (2007) considered a three-echelon supply chain having multiple retailers, while the number of distributor and manufacturer was one. Their simulation tests showed that the benefit of information sharing decreased when the correlation of customer demand between retailers was negative and it was mainly due to the risk pooling effect.

Feng (2012) constructed a simulation model based on the system dynamics approach so as to represent a three-level supply chain which was composed of a supplier, a manufacturer, a distributor and a retailer. Based on the results of simulation test, the author concluded that the supply chain with information sharing was more stable than the supply chain without information sharing, although there were fluctuations in the customer demand.

Costantino et al. (2014) considered partial information sharing where customer demand information was shared with only some part of players. That is, some players in the supply chain were aware of the customer demand, whereas others were not. They found that the whole information sharing showed better performance than the partial information sharing. Moreover, they concluded that the improvement of accuracy of demand forecasting could not replace the benefit of information sharing.

Costantino et al. (2015) considered the effect of ordering policy to the benefit of information sharing. They suggested the slow information sharing policy where the ordering quantity was divided by two parts; the actual customer demand and the adjustment of inventory position. They proved its effectiveness by performing the simulation study with various demand patterns.

Jin (2017) analyzed the effect of lead time on the benefit of information sharing by use of the system dynamics based simulation model. It was found that reducing the retailer's lead time was more important than reducing the supplier's lead time. And it was also found that reducing the variability of the lead time was more important than reducing the length of the lead time.

Even though many researches on the demand information sharing have already been published, there are still rooms for new researches because the business environment is composed of complex elements. Therefore, in this paper, various business environments which are rarely mentioned in the previous researches such as the length of ordering cycle, 
the maximum size of ordering quantity, backlog versus lost sales and the type of information shared would be taken into consideration.

Several researches on the length of ordering cycle (Bischak et al., 2014; Eruguz et al., 2014), the ordering quantity (Hsu \& Teng, 2017; Kim et al. 2011), backlog versus lost sales (Gayona et al., 2016; Singha et al., 2017) and type of information (Feng, 2012; Jin, 2017) have been published. However, it is difficult to find researches dealing with the effect of the above mentioned factors on the benefit of demand information sharing. This lack of research motivated the author and this paper deals with the simulation study so as to find the effect of the length of ordering cycle, the maximum ordering quantity, backlog versus lost sales, and type of information on the benefit of information sharing. The results of the simulation test might be useful to the company considering the adoption of some collaborative inventory management policies in the similar business environment.

\section{Simulation Model}

In this research, system dynamics approach is used to model the considered supply chain. System dynamics is defined as a perspective and set of conceptual tools that enable us to understand the structure and dynamics of complex systems (Sternman, 2000). It is useful in monitoring the long term effects of decision or policy as well as understanding the considered complex system.

System dynamics is known to be effective in modeling the complex system having feedback structure. And it has been used in many researches so as to model the considered supply chain. (Sternman, 2000; Christina, 2004; Feng, 2012; Jin, 2017)

\subsection{Model Overview}

This paper considers a two-echelon supply chain which consists of a retailer and a vendor. Outside the supply chain, a customer and a manufacturer are considered.

In this supply chain, the customer places an order with the retailer every day and the retailer responds to the daily customer demand with its on-hand inventory. If the retailer does not have enough on-hand inventories, customer demands exceeding retailer's on-hand inventory level are assumed to be lost. The retailer also places a replenishment order with the vendor to maintain its inventory level. The vendor satisfies the retailer's replenishment order with its on-hand inventory. If the replenishment order of the retailer is greater than the on-hand inventory level of the vendor, the excess of the replenishment order is assumed to be backlogged and replenished as soon as possible. The vendor places a replenishment order with the manufacturer and the manufacturer delivers the products after the predetermined fixed lead time. Capacities of the manufacturer and the transportation are assumed to be unlimited.

The distribution of customer demand is one of the most important factors in developing simulation model for the supply chain because it is the main source of uncertainty in the system. Therefore, various stochastic processes such as the normal distribution (Costantino et al., 2015), the gamma distribution (Sari, 2007) and the triangular distribution (Cho, 2011) have been considered to represent the customer demand. However, the most widely used one is the autoregressive process $\mathrm{AR}(1)$ (Lee et al., 2000; Sabitha et al., 2016). The most peculiar feature of $A R(1)$ process is that customer demand is dependent on the demand of the previous time period. Therefore, the behavior of customer demand in terms of time period can be represented by the auto regressive model. Like other previous researches, this paper assumes that the customer demand at time period $t, D_{t}$, follows $\mathrm{AR}(1)$ process as follows; (Lee et al., 2000)

$$
D_{t}=\mu+\rho D_{t-1}+\varepsilon_{t}, \quad|\rho|<1
$$

where $\mu$ is a non-negative constant, $\rho$ is an autocorrelation coefficient and $\varepsilon_{t}$ is a white noise process following the normal distribution with mean 0 and variance $\sigma^{2}$.

Another important factor to describe the supply chain is the ordering policy of each player in the supply chain. Even though lots of ordering policies have been considered in the literature, the most widely used one is the orderup-to (OUT) policy. In OUT policy, the ordering quantity at time period $t, O_{t}$, is calculated as follows; (Costantino et al., 2015)

$$
O_{t}=S_{t}-I P_{t}
$$


where $S_{t}$ means the target inventory level and $I P$, means the inventory position at time period $t$.

Target inventory level, $S_{t}$, is calculated as the sum of the average demand and the safety stock during the lead time. The level of safety stock depends on the uncertainty of demand and the required service level. To avoid unnecessary discussion on the service level, a common approach to decide the level of safety stock is to add a multiple of forecasted demand (Dejonckheere et al., 2004; Cho, 2011; Costantino et al., 2015). Inventory position, $I P_{t}$, consists of the on-hand inventory at time $t$ and the on-order inventory (or pipeline inventory, WIP) at time $t$. In OUT policy, ordering quantities are periodically calculated by use of demand forecast. Since the ordering quantity is dependent on the demand, OUT policy is sometimes called as a pull type ordering policy. Moreover, it is well known that the OUT policy minimizes the total discounted holding and shortage costs over the infinite time horizon (Lee et al., 2000). Therefore, in this paper, it is assumed that retailer and vendor place their replenishment orders according to the OUT policy.

\subsection{Stock-and-Flow Diagram}

For the comparison, two types of simulation models were developed; a supply chain without information sharing (SC-NIS) and a supply chain with information sharing (SC-IS). The considered supply chains were modeled with Vensim PLE version 6.4. Fig. 1 and Fig. 2 show the stock-and-flow diagrams which were developed to represent the considered supply chains. In these figures, the level variables are displayed in the rectangular box and the auxiliary variables are described in the clear box. Variables in the bracket are shadow variables.

Customer Demand represents the volume of daily customer demand following $A R(1)$ process. Retailer's Sales is calculated as the minimum value of Customer Demand and Retailer's INV which represents the level of on-hand inventory at retailer site. If Customer Demand is larger than Retailer's INV, then the excess is recorded at Retailer's Stockout. Retailer's WIP represents the on-order inventory of retailer, that is, the amount of products shipped by vendor but not yet arrived. Forecast of Customer Demand is used to calculate Retailer's Order and obtained by exponentially smoothing the daily customer demand. Retailer's Order is calculated by

\section{Fig. 1: Stock and flow diagram of SC-NIS}

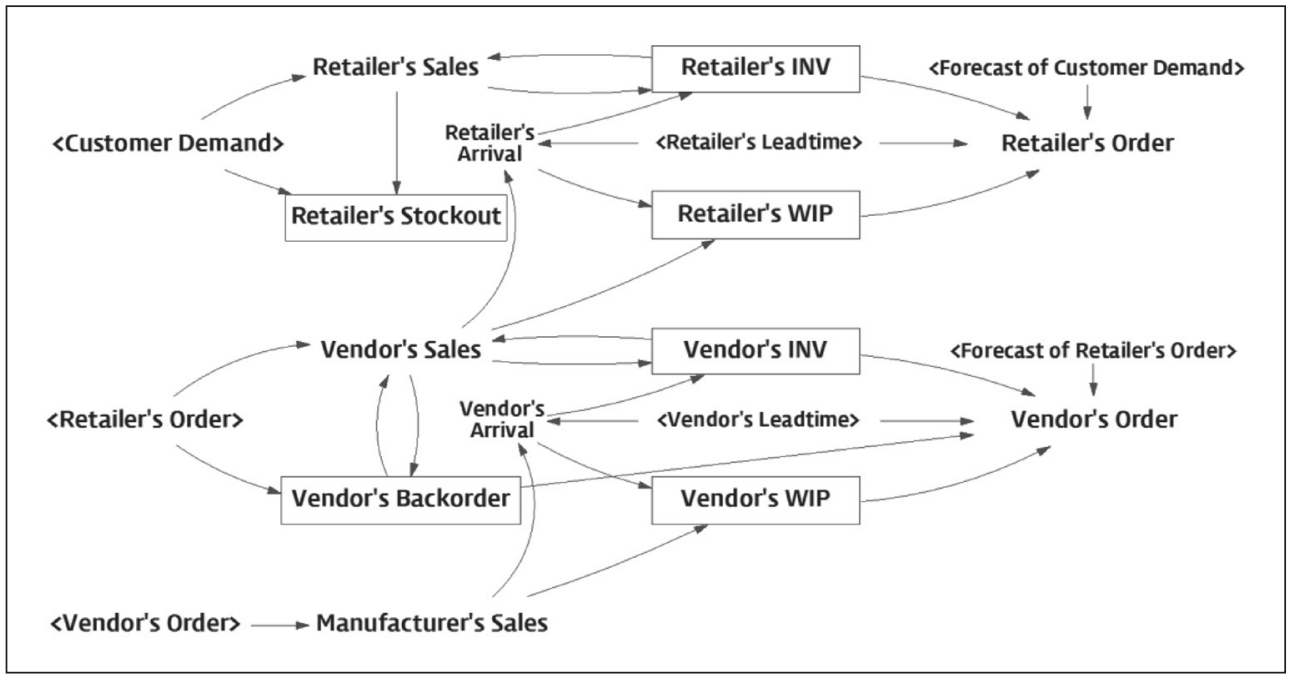




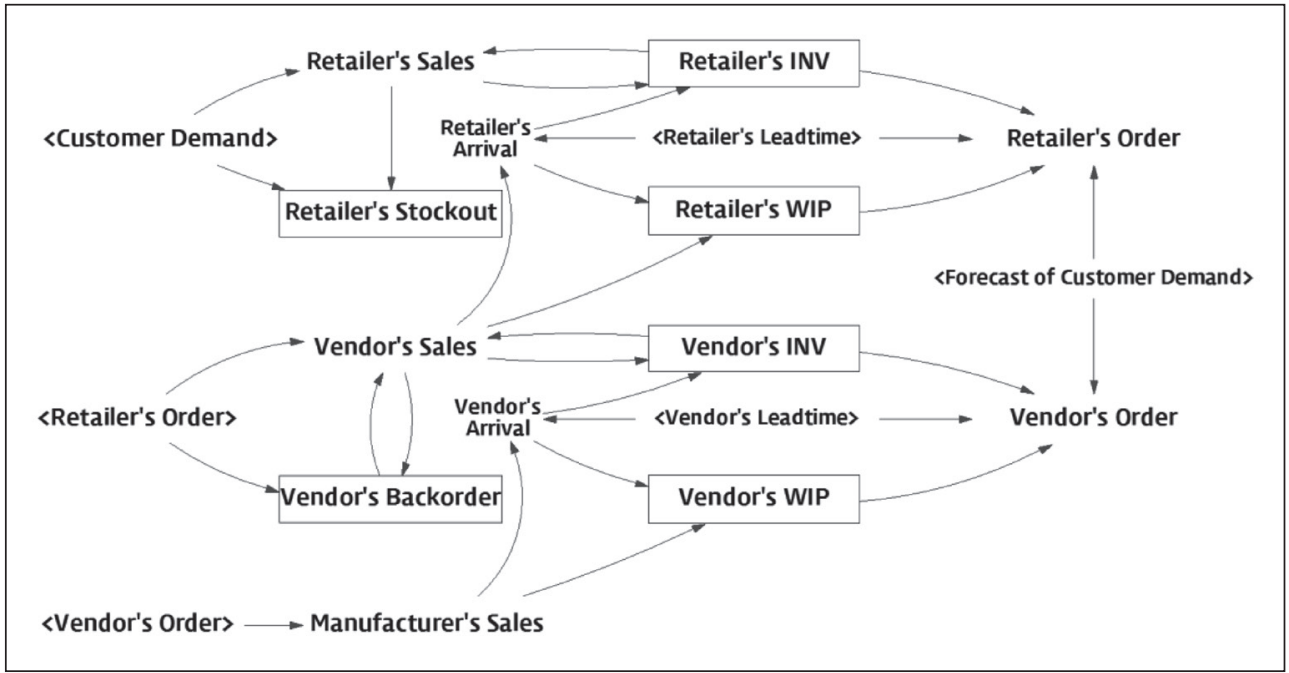

Source: own

Eq. (2). The target inventory level of the retailer's replenishment order is calculated by considering Retailer's Leadtime and Forecast of Customer Demand and the inventory position is calculated by summarizing Retailer's INV and Retailer's WIP.

Vendor's Sales is calculated as the minimum value of Vendor's INV and the sum of Retailer's Order and Vendor's Backorder which represents the accumulated unsatisfied retailer's order. Vendor's Inventory and Vendor's WIP are updated in the same way as Retailer's Inventory and Retailer's WIP. The difference between SC-NIS and SC-IS comes from the input variables of Vendor's Order. In SC-NIS, Forecast of Retailer's Order, which is obtained by exponentially smoothing Retailer's Order, is used to calculate the target inventory level of Vendor's Order. And the inventory position of Vendor's Order is calculated by subtracting Vendor's Backorder from the sum of Vendor's Inventory and Vendor's WIP. On the other hand, Forecast of Customer Demand, which was used to calculate Retailer's Order, is also used to calculate the target inventory level of Vendor's Order in SC-IS. Moreover, unlike SC-NIS, Vendor's Backorder is not taken into account in calculating the inventory position of Vendor's Order in SC-IS so as to apply the echelon stock policy of information sharing.

Since the capacity of manufacturer is assumed to be unlimited, Manufacturer's Sales is the same to Vendor's Order and it is delivered to the vendor after the predetermined lead time.

\section{Experimental Results}

\subsection{Experimental Design}

To evaluate the effect of demand information sharing with the system dynamics models described in Section 2, the experimental tests considering various business environments were performed. This section describes the overall environment for the simulation test.

For model setting, the time step is set to 1 day and the final time is set to 800 days. Test results of the first 100 days and the last 100 days are not considered in the analysis. Benefit of demand information sharing is measured with respect to the bullwhip effect, the level of inventory in the whole supply chain and the stockout rate at retailer. For the test under various business environments, the following scenarios are developed and the data sets based on the developed scenarios are randomly generated by changing the random seed of white noise process $\varepsilon_{t}$ of customer demand. 
The first scenario is to monitor the benefit of demand information sharing when the review period of ordering policy is changed. In this scenario, the review period of ordering is changed from one day to four days.

The second scenario is to compare the effect of maximum ordering quantity. That is, in this scenario, the ordering quantity of retailer and vendor cannot exceed the maximum ordering quantity, $\bar{O}$, which is set as follows:

$$
\bar{O}=C V \times L T \times \frac{\mu}{(1-\rho)}
$$

where $L T$ is the length of lead time and $C V$ is the ordering coefficient value.

In the second scenario, five different levels of the maximum ordering quantity are considered by changing the ordering coefficient value, $C V$. In each level, $C V$ is set to $0.6,0.9$, $1.2,1.5$ and infinite value. That is, if $\mathrm{CV}$ is set to 0.9 , LT to 5 days, $\mu$ to 100 and $\rho$ to 0.7 , then the maximum ordering quantity is 1,500 .

The third scenario is to compare the policies on handling the shortage of vendor's inventory. That is, in the first case, the shortage of vendor's inventory is backlogged as described in Section 2. However, in the second case, the shortage of vendor's inventory is treated to be lost as in the retailer's site. In some industries, vendor may deliver the unsatisfied retailer's order in the near future. However, in other industries, it may not be allowed.

The fourth scenario is to compare the types of information shared by retailer and vendor. In the base model described in Section 2, the retailer shares the customer demand with the vendor. However, in some cases, the amount of retailer's sales is shared instead of the actual customer demand (Feng, 2012). The difference between two types of information is the amount of stockout at the retailer. That is, usually, the retailer's sales are less than or equal to the customer demand.

For the fourth scenario, two sub scenarios are developed based on the main reason of the retailer's stockout. The first sub scenario assumes that the retailer's stockout is mainly due to the insufficient safety stock at the retailer site. And the second sub scenario assumes that the retailer's stockout is mainly due to the customer's exceptional peak demand.

\section{Tab. 1: Test scenarios}

\begin{tabular}{|c|c|c|}
\hline Considered Factor & Variables Setting & Notation \\
\hline \multirow{4}{*}{ 1. Length of review period } & Review period is one day & SN1-1 \\
\hline & Review period is 2 days & SN1-2 \\
\hline & Review period is 3 days & SN1-3 \\
\hline & Review period is 4 days & SN1-4 \\
\hline \multirow{5}{*}{ 2. Maximum ordering quantity } & $\mathrm{CV}$ is set to 0.6 & SN2-1 \\
\hline & CV is set to 0.9 & SN2-2 \\
\hline & $\mathrm{CV}$ is set to 1.2 & SN2-3 \\
\hline & $\mathrm{CV}$ is set to 1.5 & SN2-4 \\
\hline & $\mathrm{CV}$ is set to infinite value & SN2-5 \\
\hline \multirow{2}{*}{ 3. Policy on the shortage of vendor's inventory } & Shortage is backlogged & SN3-1 \\
\hline & Shortage is lost & SN3-2 \\
\hline \multicolumn{3}{|l|}{ 4. Type of shared information } \\
\hline \multirow{2}{*}{ 4-1. Stockout due to insufficient safety stock } & Customer Demand is shared & SN4-1-1 \\
\hline & Retailer's Sales is shared & SN4-1-2 \\
\hline \multirow{2}{*}{ 4-2. Stockout due to exceptional customer demand } & Customer Demand is shared & SN4-2-1 \\
\hline & Retailer's Sales is shared & SN4-2-2 \\
\hline
\end{tabular}


In this simulation test, the partial factorial analytical method is adopted. That is, to analyze the effect of one factor, the value of specific factor is changed while others remain the same. This method simplifies the analysis process and helps us to concentrate on the effect of the design factor itself. Even though it does not describe whole complicated situations, it still provides plentiful insights to researchers. (Zhang \& Zhang, 2007)

\subsection{Result Analysis of Base Model}

Prior to the simulation test based on the developed scenarios, experimental tests with the base model were performed. Experimental test with the base model might provide an overall insight on the benefit of demand information sharing as well as it can be used as a baseline result to be compared to each scenario.

In the base model, customer demands are set to follow the autoregressive process where $\mu$ is 100 and $\rho$ is 0.7 so that the average daily customer demand is 333. Lead times of retailer and vendor are set to five days. For reliable tests, ten different data sets are randomly generated by changing the random seed of white noise process $\varepsilon_{t}$ following the normal distribution. The averaged test results are summarized in Fig. 3.

Performance of supply chain is measured in terms of the bullwhip effect, the inventory

\section{Fig. 3: Test result of base model}

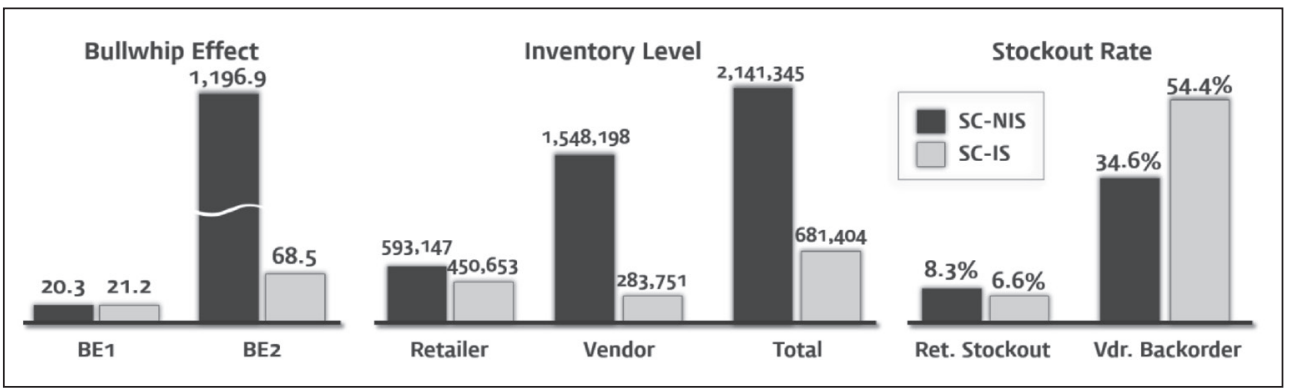

Source: own

level and the stockout rate. The bullwhip effect can be measured as the ratio of the variances of ordering quantity. BE1 is the ratio of the variance of retailer's replenishment order to the variance of customer demand, while BE2 is the ratio of the variance of vendor's replenishment order to the variance of customer demand. For example, in SC-NIS, the variance of customer demand is $10,874.5$ and the variance of retailer's replenishment order is 220,312.7 and the variance of vendor's replenishment order is $12,982,830.8$ so that BE1 of SC-NIS is 20.3 and BE2 of SC-NIS is 1,196.9.

In this research, the bullwhip effect of supply chain is measured by BE2 so as to measure the benefit of information sharing. Therefore, test results show that demand information sharing reduces the bullwhip effect, which is the expected result. More interesting result is that BE1 in SC-IS is slightly higher than BE1 in
SC-NIS. It means that the variance of retailer's ordering quantity does not decrease even though demand information is shared. It might be inferred that shared information has an effect mostly on the vendor and its downstream player.

In terms of inventory level, the vendor's inventory level decreases by $81.7 \%$ when demand information is shared, while the retailer's inventory level decreases by $24.0 \%$. It means that most of the benefits in terms of inventory reduction are enjoyed by vendor rather than retailer. It corresponds to the result of Lee et al. (2000) where they claimed that some incentives in terms of financial and operational scheme should be provided to retailer by vendor to share customer demand.

Regarding the stockout rate against customer demand, SC-IS shows $6.6 \%$, a reduction of $20.3 \%$ compared to SC-NIS. 
That is, demand information sharing also brings the improvement of stockout rate as well as inventory reduction. In both cases, the backorder rate of vendor is higher than the stockout rate of retailer. It might be inferred that higher variance of retailer's order (compared to the variance of customer demand) brings larger uncertainty to the vendor. An interesting result is that the backorder rate at SC-IS is $54.4 \%$ which is higher than that of SC-NIS. This might be explained as follows.

In SC-NIS, the vendor reacts to the retailer's replenishment order itself. However, in SC-IS, the vendor reacts to the customer demand, not to the retailer's replenishment order. Therefore, the discrepancy between the customer demand and the retailer's replenishment order brings larger backorder against the retailer's replenishment order when the customer demand information is shared.
In summary, from the experimental test results with the base model, we can conclude that demand information sharing provides a high degree of flexibility in the supply chain by satisfying customer demand more accurately with smaller inventory level. In addition, the flexibility arises mostly from the vendor's behavior because information on the customer demand provides more visibility to the vendor rather than the retailer.

\subsection{Result Analysis of Scenario Test}

This section describes the results of the experimental tests based on the scenarios mentioned in Section 3.1. In the first scenario, the review period of replenishment ordering is changed from one day to four days and the following figure summarizes the experimental test results.

\section{Fig. 4: Improvement ratio of demand information sharing in Scenario 1}

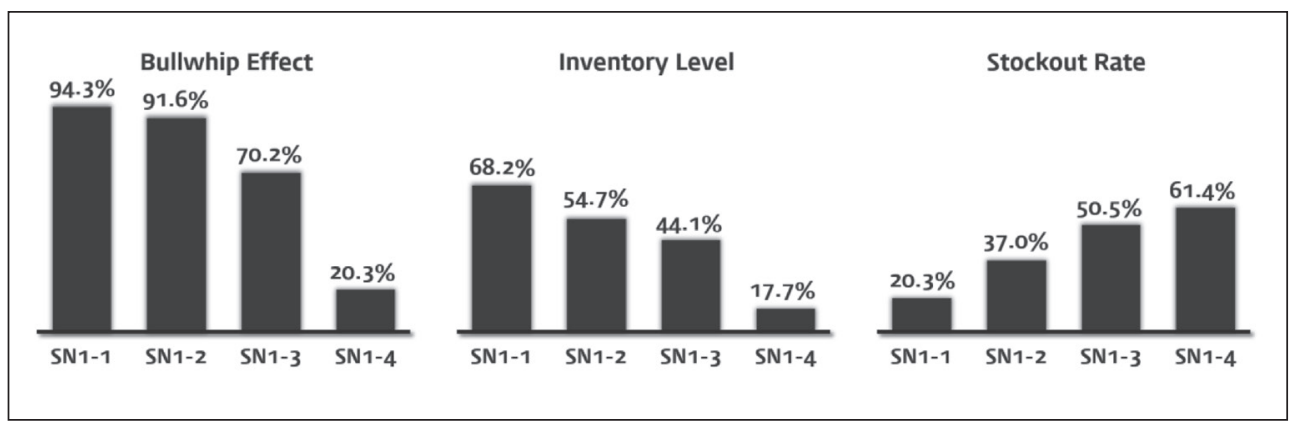

Source: own

Fig. 4 shows the improvement ratio of SC-IS compared to SC-NIS. That is, when the review period of ordering is set to four days, SC-IS shows $20.3 \%$ smaller bullwhip effect, $17.7 \%$ lower level of inventory, and $61.4 \%$ lower stockout rate than SC-NIS.

In terms of the bullwhip effect, the performance improvement brought by demand information sharing reduces as the review period increases. The increase of review period means the increase of uncertainty in demand and supply across the whole supply chain. The increase of uncertainty in the supply chain also increases the bullwhip effect regardless of demand information sharing. However, the increasing rate of SC-IS is steeper than that of SC-NIS. It means that the benefit of demand information sharing in terms of the bullwhip effect decreases as the review period increases.

The benefit of demand information sharing in terms of inventory level also decreases as the review period increases. This is the expected result since the level of inventory tends to depend on the level of bullwhip effect. With respect to the stockout rate, both SC-IS and SC-NIS show worse performance as the review period increases. However, the improvement ratio of demand information sharing increases since the increasing rate of SC-NIS is steeper than that of SC-IS. That is, the stockout rate of 


\section{Fig. 5: Improvement ratio of demand information sharing in Scenario 2}

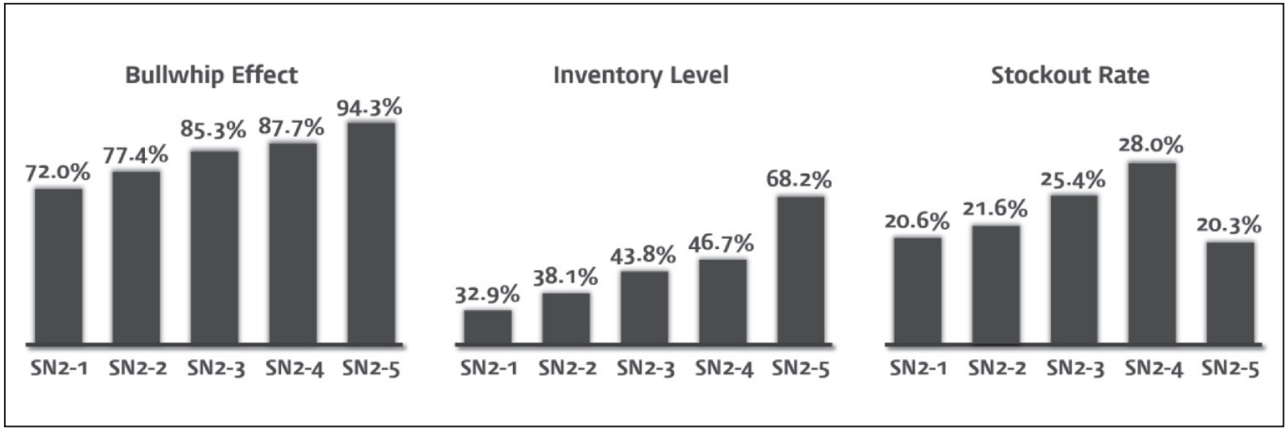

Source: own

SC-NIS increased from $8.3 \%$ when the review period was one day to $20.0 \%$ when the review period was four days. On the other hand, the stock out rate of SC-IS increased from $6.6 \%$ when the review period was one day to $7.7 \%$ when the review period was four days.
In summary, from the test result of Scenario 1, we can conclude that the increase of review period results in a reduction of the inventory level improvement effect of demand information sharing by reducing the benefit of bullwhip effect improvement. Therefore, it might be claimed

\section{Fig. 6: Vendor's ordering quantities in Scenario 2}

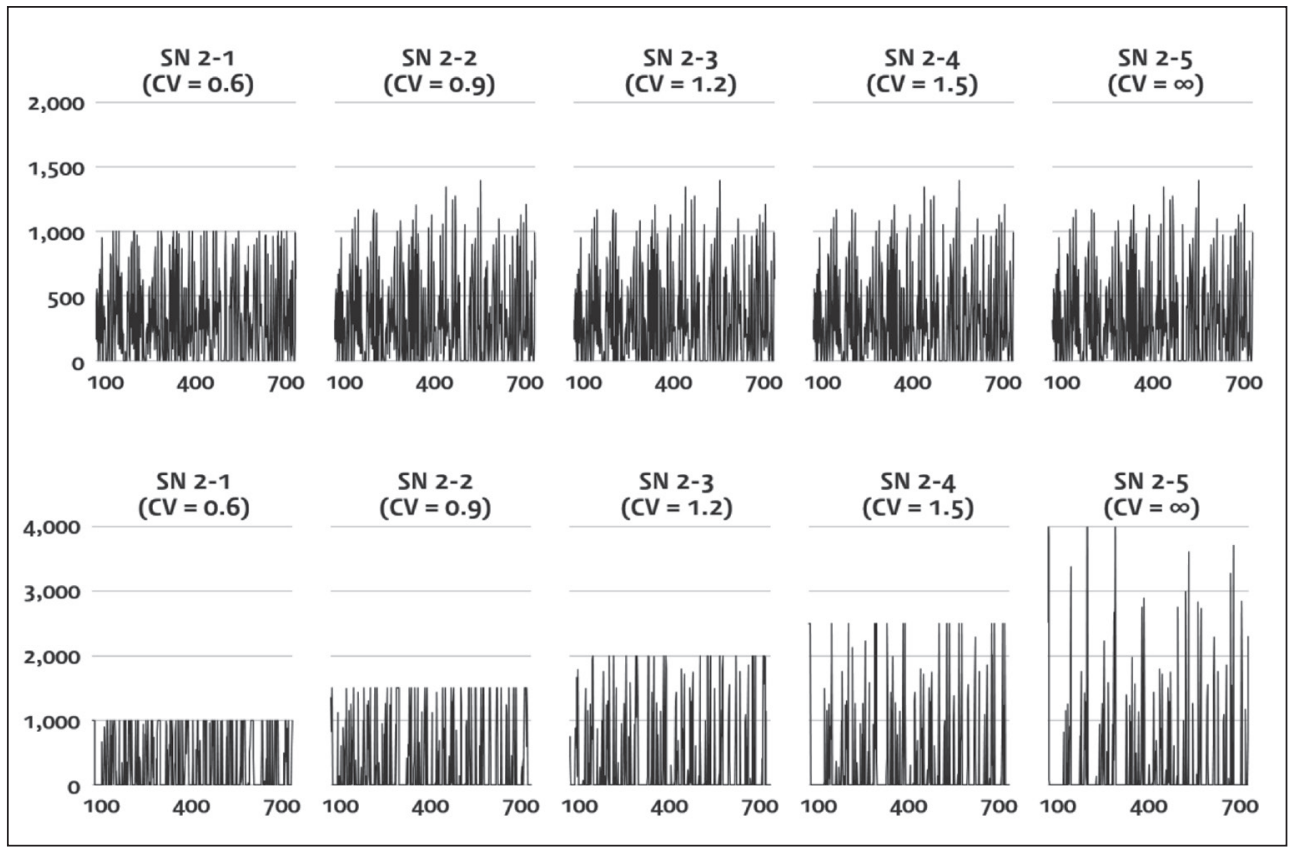

Source: own 
that, when a company wants to share demand information with its partners, it is needed to shorten the review period of replenishment ordering in order to maximize the benefit of demand information sharing.

In the second scenario, the effect of maximum ordering quantity of retailer and vendor on the demand information sharing is tested.

Fig. 5 shows the change of the improvement ratio of SC-IS compared to SC-NIS while the ordering coefficient value is changed. With respect to the bullwhip effect, a strict constraint on the maximum ordering quantity (that is, a low $C V$ value) reduces the improvement ratio of demand information sharing. Decrease of the improvement ratio on the bullwhip effect also reduces the improvement ratio on the inventory level. However, even though the improvement ratio on the inventory level decreases as the ordering coefficient value decreases, the improvement effect on the stock out rate also decreases. Moreover, the test result of SN2-5 (unlimited ordering quantity) is different from the others with respect to the stockout rate. The different behavior of SN2-5 may be due to the fact that SC-NIS can respond to the high customer demand with unlimited ordering quantity, whereas it is difficult when the maximum ordering quantity is limited.

From the above results, it can be inferred that the benefit of demand information sharing decreases when there is a strict constraint on the maximum ordering quantity. However, an interesting finding in this test result is that the difference in performance comes mainly from SC-NIS, not from SC-IS. Fig. 6 shows the change of vendor's ordering quantity in SC-IS and SC-NIS when the ordering coefficient value changes.

In Fig. 6, the upper graph comes from SC-IS and the lower graph comes from SC-NIS. In SC-IS, there are no big differences in the vendor's ordering quantity even though the maximum ordering quantity is changed. However, in SC-NIS, the vendor's ordering quantity decreases and the frequency of ordering increases as the maximum ordering quantity decreases. Small and frequent ordering is known to be effective in reducing the bullwhip effect by preventing the order batching which is one of the main sources of the bullwhip effect (Lee et al., 1997). Therefore, the decrease of the improvement ratio in terms of the maximum ordering quantities results from the performance improvement of non-sharing environment, not from the performance degeneration of demand information sharing environment.

In summary, from the test result of Scenario 2, we can conclude that the decrease of maximum ordering quantity results in the reduction of performance improvement ratio of demand information sharing by improving the performance of SC-NIS. Therefore, it might be claimed that, when a company cannot share demand information with its partners, it is recommended to reduce the maximum ordering quantity in order to reduce the order batching effect. However, it needs to be remembered that the performance of SC-NIS did not exceed that of SC-IS even though the maximum ordering quantity was set to very small value.

In the third scenario, the shortage of vendor's on-hand inventory against the retailer's

\section{Fig. 7: Improvement ratio of demand information sharing in Scenario 3}

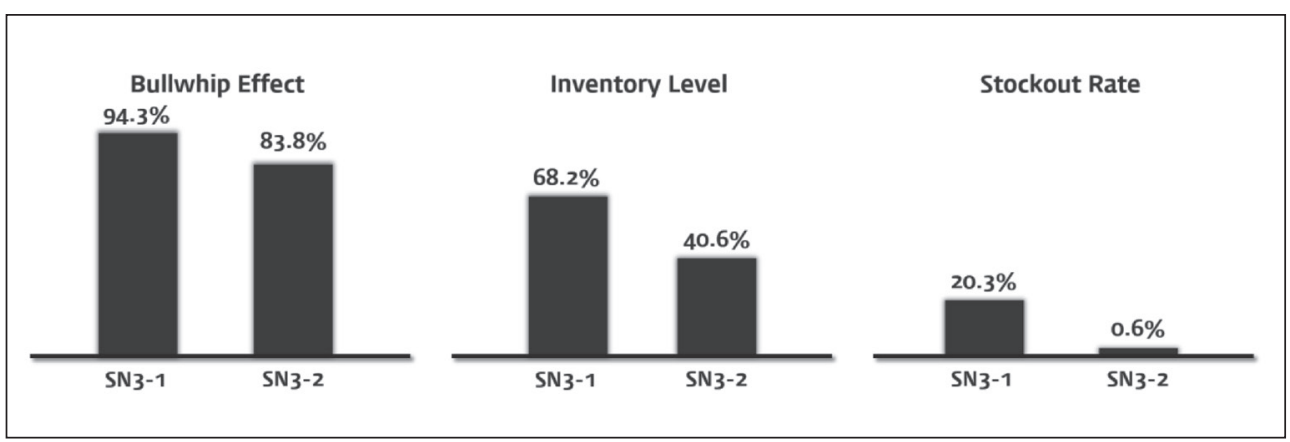


replenishment order is treated in different ways; to be lost or backlogged. In the base model, the shortage of vendor's on-hand inventory is assumed to be backlogged. Therefore, additional tests have been performed assuming that the shortage of vendor's on-hand inventory is lost.

Fig. 7 compares the benefit of demand information sharing with backlogged retailer's replenishment order and lost retailer's replenishment order. If the excessive retailer's replenishment order is assumed to be lost, the benefit of demand information sharing in terms of the bullwhip effect decreases by more than 10pp. The degeneration of the bullwhip effect also brings about the degeneration of inventory level reduction effect. Moreover, with respect to the stockout rate, there is little or no benefit of customer demand information sharing if the excessive retailer's order is lost. It corresponds to the test result of Jin (2017) where the author mentioned that the main benefit of information sharing is the reduction of inventory level rather than the reduction of stockout rate when the excessive retailer's order is treated to be lost. This might be inferred that the lost retailer's replenishment order reduces the flexibility of customer demand information sharing. However, it is worthwhile to note that the performance of demand information sharing outperforms that of the supply chain where customer demand information is not shared in both cases.

Since the degeneration of the stockout rate with the lost retailer's replenishment order is obvious, it is needed to consider how to overcome this problem. The most simple and promising method to reduce the stockout rate is to increase the safety stock level. Since there are two players (a retailer and a vendor) in the considered supply chain, safety stock level at each site can be increased. Fig. 8 shows the improvement ratio of demand information sharing when each safety stock level increases. In Fig. 8, SS1 means the same safety stock level with Fig. 7. In SS2, the safety stock level of retailer increases twofold. In SS3, the safety stock level of vendor is doubled. And in SS4, both the retailer's safety stock level and the vendor's safety stock level increase two times higher than those of SS1.

As easily anticipated, SS4 shows the biggest improvement ratio in terms of stockout rate and the lowest improvement ratio in terms of inventory level. More interesting result is to compare SS2 and SS3. With respect to all performance measures, SS2 shows better results than SS3. That is, holding additional safety stock at the retailer site is more effective than to hold additional safety stock at the vendor site. In addition, SS2 shows the biggest improvement ratio with respect to the bullwhip effect. And there are no big differences between SS2 and SS4 in terms of stockout rate, nor between SS2 and SS1 in terms of inventory level. Therefore, we may infer from the results of the above tests that increasing the safety stock at the retailer site would be desirable when the excessive retailer's replenishment order cannot be backlogged.

\section{Fig. 8: Improvement ratio of demand information sharing with respect}

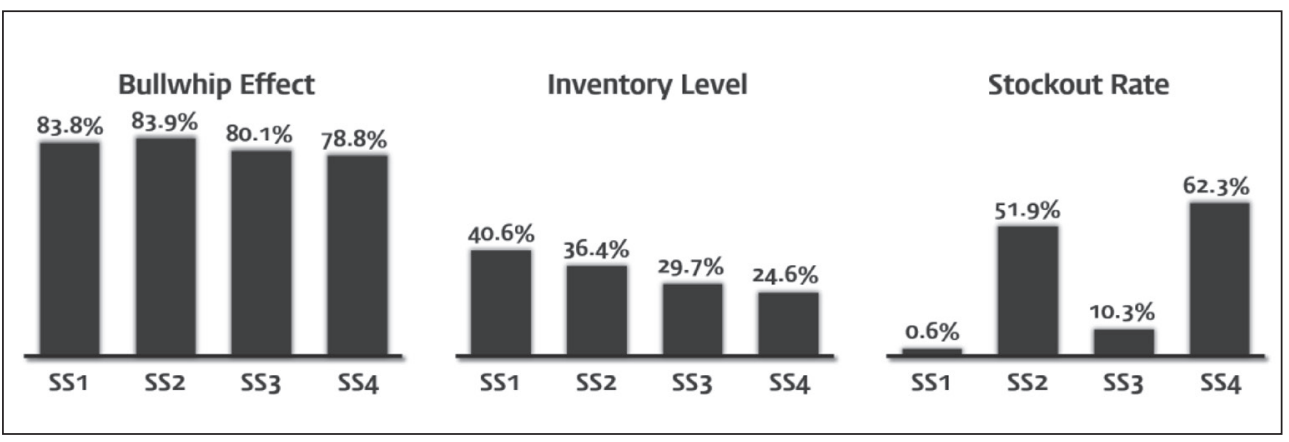




\section{Fig. 9: Improvement ratio of demand information sharing in Sub Scenario 4-1}

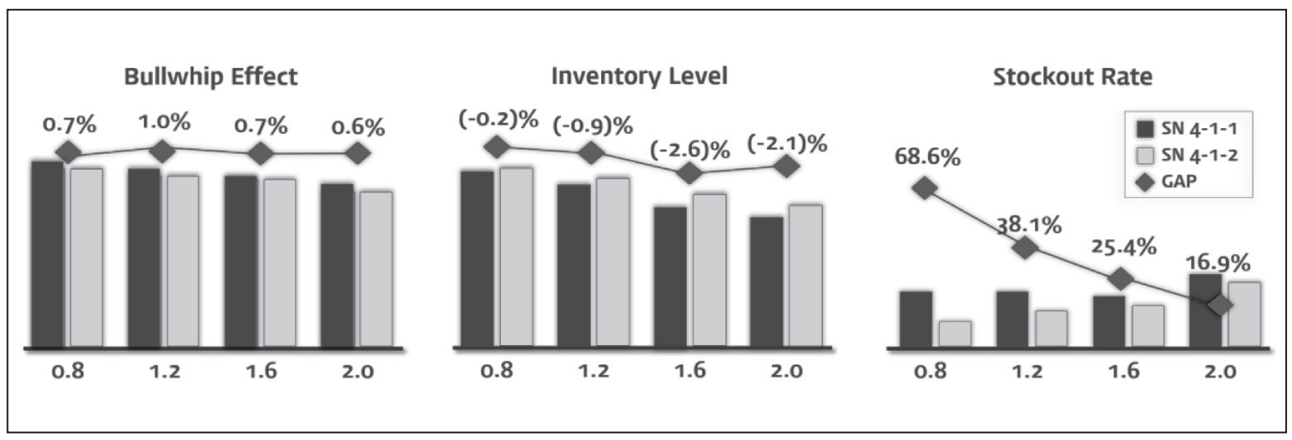

Source: own

The fourth scenario considers the impact of the types of demand information shared between retailer and vendor. As mentioned in Section 3.1, the first sub scenario assumes that the retailer's stockout is mainly due to the insufficient retailer's safety stock. For the test, the multiplication coefficient of the safety stock level at the retailer is set to $0.8,1.2,1.6$ and 2.0.

Fig. 9 shows the results of the experimental tests under the sub scenario 4-1. In Fig. 9, GAP between the performances of two types of demand information is calculated as Eq. (4) - see below.

For example, when the multiplication coefficient of the safety stock level at the retailer is set to 0.8 , the bullwhip effect of SC-NIS is $1,378.22$. If the customer demand is shared with vendor (that is, SN4-1-1), then the bullwhip effect of SC-IS (customer demand sharing) reduces to 39.26 which means $97.15 \%$ reduction of the bullwhip effect. If the retailer's sales is shared with vendor (that is, SN4-1-2), then the bullwhip effect of SC-IS (retailer's sales sharing) reduces to 49.01 , which means $96.44 \%$ reduction of the bullwhip effect. Since Improvement Ratio with Customer Demand is $97.15 \%$ and Improvement Ratio with Retailer's Sales is $96.44 \%$, then GAP is $0.7 \%$.

As anticipated, the improvement ratio with customer demand outperforms the improvement ratio with retailer's sales in terms of stockout rate, and vice versa in terms of inventory level. Among these performance measures, more peculiar trend is found in the stockout rate. In other words, as the level of safety stock decreases, the gap between customer demand and retailer's sales increases. In terms of inventory level, the gap between customer demand and retailer's sales is not as big as the stockout rate. Moreover, the gap decreases as the level of safety stock decreases.

The second sub scenario assumes that the retailer's stockout is mainly due to the customer's exceptional peak demand. Unexpected peak demand from the customer may happen for various reasons such as promotion and price fluctuation, which have been pointed as one of the main reasons of the bullwhip effect (Lee et al., 1997). In this sub scenario, it is assumed that there are promotions every 30 days and customer demand is amplified by the inflation factor (IF) as Fig. 10.

Fig. 11 shows the results of the experimental tests under the sub scenario 4-2. The improvement ratio with customer demand outperforms the improvement ratio with retailer's sales in terms of stockout rate, and vice versa in terms of inventory level. However, as the inflation factor increases, the performance of customer demand declines sharply, so that the improvement ratio with retailer's sales outperforms the improvement ratio with customer demand in terms of all performance measures. It might be inferred

(Improvement Ratio with Customer Demand - Improvement Ratio with Retailer's Sales)

Improvement Ratio with Retailer's Sales 


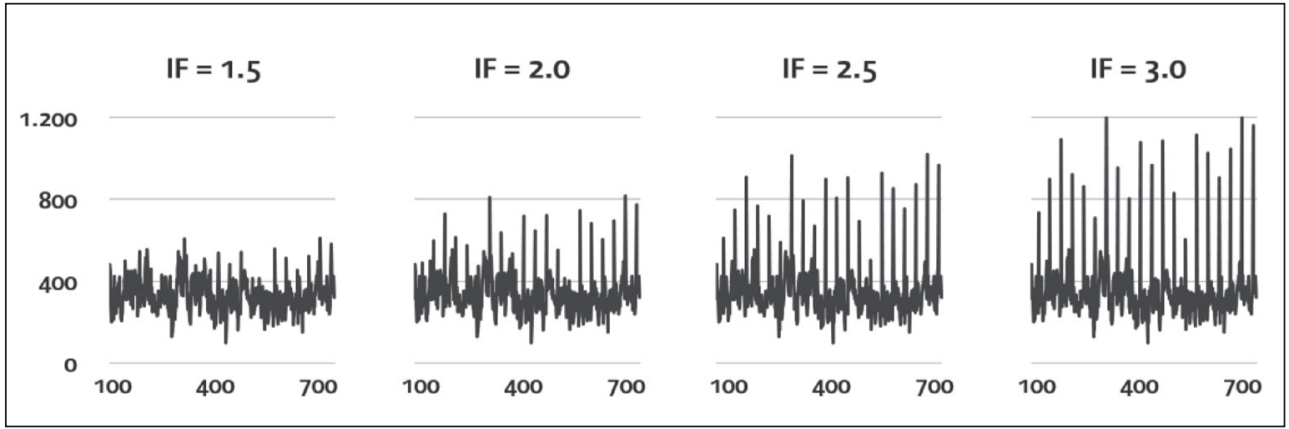

Source: own

\section{Fig. 11: Improvement ratio of demand information sharing in Sub Scenario 4-2}
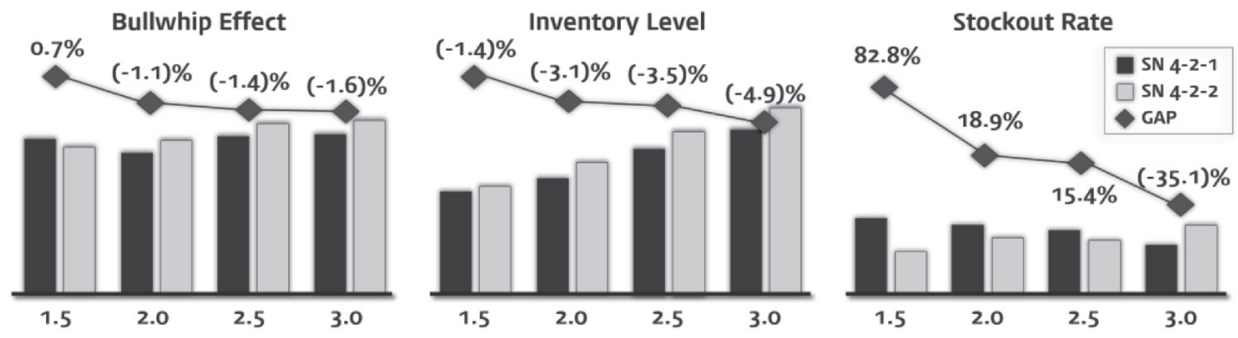

Source: own

\section{Tab. 2: Summary of test result}

\begin{tabular}{c|l} 
Test Result & \multicolumn{1}{c}{ Managerial Implication } \\
\hline Fig. 3 & $\begin{array}{l}\text { Information sharing between companies increases supply chain's flexibility so that } \\
\text { higher customer service is possible with lower inventory level. However, the benefit } \\
\text { in terms of inventory reduction comes mainly from the vendor, not from the retailer. }\end{array}$ \\
\hline Fig. 4 & $\begin{array}{l}\text { Increasing the review period of ordering process results in a reduction in the inventory } \\
\text { level improvement effect. Therefore, it is recommended to keep the review period } \\
\text { as short as possible. }\end{array}$ \\
\hline Fig. 5 & $\begin{array}{l}\text { If the customer demand information is not shared, reducing the maximum ordering quantity } \\
\text { may improve the inventory level and the stockout rate by reducing the bullwhip effect. }\end{array}$ \\
\hline Fig. 6 & $\begin{array}{l}\text { If the shortage of vendor's on-hand inventory against the retailer's replenishment order is } \\
\text { treated to be lost, it is recommended to increase the safety stock level at the retailer site. }\end{array}$ \\
\hline Fig. 7 & $\begin{array}{l}\text { In general cases, sharing customer demand is expected to be better than sharing } \\
\text { retailer's sales. However, a data cleaning process which eliminates the effect of artificial } \\
\text { events is essential to share the customer demand information. }\end{array}$ \\
\hline Fig. 9 & Fig. 11
\end{tabular}


that the information of retailer's sales tends to smooth the large unexpected change in customer demand. However, if the information of customer demand is shared, the stability of future demand forecast is degenerated because of the large unexpected change in customer demand. Therefore, if a company wants to share customer demand with its partners, a data cleaning process is required to eliminate the effect of artificial events such as price fluctuation and promotion.

\subsection{Managerial Implication}

This section summarizes some interesting findings from the above mentioned simulation test and their managerial implications are listed in Tab. 2.

\section{Conclusions}

The purpose of this research is to analyze the benefits of demand information sharing under various business environments. It is well known that the bullwhip effect in a supply chain can be reduced by sharing useful information such as customer demand and inventory level with other players in the supply chain. However, the benefit of information sharing may vary as the business environment of the supply chain is changed. Therefore, it is needed to find an appropriate environment for information sharing.

In this research, two simulation models based on the system dynamics approach were developed to represent the supply chain with information sharing (SC-IS) and noninformation sharing (SC-NIS). The system dynamics approach models each component of the considered system as a numerical form and quantifies the dynamics of its relationships over time. Therefore, it might be an appropriate approach to monitoring the long term performance of demand information sharing.

To find the benefit of demand information sharing under various business environments, four different scenarios considering the review period of ordering policy, the maximum ordering quantity, the policy on the shortage of vendor's on-hand inventory and the type of shared information were developed. The results of the experimental tests were analyzed with respect to the bullwhip effect, the inventory level in the whole supply chain and the stockout rate of the retailer. Some of the experimental test results corresponded to the results of the previous research and there were also some interesting findings. The new findings were analyzed and their managerial implications were discussed. These managerial implications might be useful for a company considering a collaborative inventory management policy based on the demand information sharing with its partners.

Even though this paper may provide some useful insights into the benefits of demand information sharing under various business environments, there are also some limitations in the research. First, the supply chain considered in this research is assumed to have two echelons and single player at each echelon. Even though the overall effect of supply chain can be understood with this simple structure, the effect of multiple players in the same echelon cannot be analyzed. Therefore, future research considering more complex supply chain would be useful in extending the result of this paper. Second, even though some various business environments were considered in this research, there are still a lot of business environments to be considered, such as the capacity of each player, types of commodity and industries. These are also interesting research topics which can be done in the near future.

This work was supported by Hannam University Research Fund.

\section{References}

Bischak, D. P., Robb, D. J., Silver, E. A., \& Blackburn, J. D. (2014). Analysis and management of periodic review, Order-Up-To level inventory systems with order crossover. Production and Operations Management, 23(5), 762-772. https://doi.org/10.1111/poms.12072.

Chen, F., Drezner, Z., Ryan, J. K., \& SimchiLevi, D. (2000). Quantifying the bullwhip effect in a simple supply chain: the impact of forecasting, lead times and information. Management Science, 46(3), 436-443. https://doi.org/10.1287/mnsc.46.3.436.12069.

Cho, M. (2011). Impact of smoothed replenishment ordering policy on the performance measures in supply chain. Journal of Korean Society of Simulation, 20(2), 19-27. https://doi.org/10.9709/JKSS.2011.20.2.019.

Christina, T. S. (2004). System dynamics model as a decision support tool for inventory management improvement (Doctoral dissertation). Delft University of Technology, Netherlands.

Costantino, F., Gravio, G. D., Shaban, A., \& Tronci, M. (2014). The impact of information 
sharing and inventory control coordination on supply chain performances. Computers \& Industrial Engineering, 76, 292-306. https://doi. org/10.1016/j.cie.2014.08.006.

Costantino, F., Gravio, G. D., Shaban, A., \& Tronci, M. (2015). The impact of information sharing on ordering policies to improve supply chain performances. Computers \& Industrial Engineering, 82, 127-142. https://doi. org/10.1016/j.cie.2015.01.024.

Dejonckheere, J., Disney, S. M., Lambrecht, M. R., \& Towill, D. R. (2004). The impact of information enrichment on the bullwhip effect in supply chains: A control engineering perspective. European Journal of Operational Research, 153(3), 727-750. https://doi. org/10.1016/S0377-2217(02)00808-1.

Eruguz, A. S., Jemai, Z., Sahin, E., \& Dallery, Y. (2014). Optimising reorder intervals and order-up-to levels in guaranteed service supply chains. International Journal of Production Research, 52(1), 149-164. https://doi.org/10.10 80/00207543.2013.831188.

Feng, Y. (2012). System dynamics modeling for supply chain information sharing. Physics Procedia, 25, 1463-1469. https://doi. org/10.1016/j.phpro.2012.03.263.

Gayon, J., Massonnet, G., Rapine, C., \& Stauffer, G. (2016). Constant approximation algorithms for the one warehouse multiple retailers problem with backlog or lost-sales. European Journal of Operational Research, 250, 155-163. https://doi.org/10.1016/j.ejor.2015.10.054.

Hsu, P., \& Teng, H. (2017). Optimal ordering quantities with stochastic demand under deterioration and amelioration. In L. Yongqiang, A. Hunjet, \& A. Roncevic (Eds.), Proceeding of the 20th International Scientific Conference on Economic and Social Development (pp.103-110). Prague, Czech Republic.

Jin, H. (2017). Analysis of leadtime effects on VMI system: System dynamics approach. International Journal of Applied Management Science, 9(1), 38-54. https://doi.org/10.1504/ IJAMS.2017.082415.

Lee, H. L., Padmanabhan, V., \& Whang, S. (1997). The bullwhip effect in supply chains. Sloan Management Review, 38(3), 93-102.

Lee, H. L., So, K. C., \& Tang, C. S. (2000). The value of Information sharing in a two-level supply chain. Management Science, 46(5), 626-643. https://doi.org/10.1287/mnsc.46.5.626.12047.

Kim, H., Lu, J., Kvam, P. H., \& Tsao, Y. (2011). Ordering quantity decisions considering uncertainty in supply-chain logistics operations. International Journal of Production Economics, 134(1), 16-27. https://doi.org/10.1016/j. ijpe.2011.02.017.

Sabitha, D., Rajendran, C., Kalpakam, S., \& Ziegler, H. (2016). The value of information sharing in a serial supply chain with $\operatorname{AR}(1)$ demand and non-zero replenishment lead times. European Journal of Operational Research, 255(3), 758-777. https://doi. org/10.1016/j.ejor.2016.05.016.

Sari, K. (2007). Exploring the benefits of vendor managed inventory. International Journal of Physical Distribution \& Logistics Management, 37(7), 529-545. https://doi. org/10.1108/09600030710776464.

Singha, K., Buddhakulsomsiri, J., \& Parthanadee, P. (2017). Mathematical model of $(R, Q)$ inventory policy under limited storage space for continuous and periodic review policies with backlog and lost sales. Mathematical Problems in Engineering, 2017, 1-9. https://doi.org/10.1155/2017/4391970.

Sternman, J. D. (2000). Business dynamics: Systems thinking and modeling for a complex world. New York: McGraw-Hill.

Thomas, R., \& Esper, T. (2010). Exploring relational asymmetry in supply chains: the retailer's perspective. International Journal of Physical Distribution \& Logistics Management, 40(6), 475-494. https://doi. org/10.1108/09600031011062209.

Yigitbasioglu, O. (2010). Information sharing with key suppliers: a transaction cost theory perspective. International Journal of Physical Distribution \& Logistics Management, 40(7), 550-578. https://doi. org/10.1108/09600031011072000.

Yu, Z., Yan, H., \& Cheng, E. (2001). Benefits of information sharing with supply chain partnerships. Industrial Management \& Data Systems, 101(3), 114-119. https://doi. org/10.1108/02635570110386625.

Zhang, C., \& Zhang, C. (2007). Design and simulation of demand information sharing in a supply chain. Simulation Modelling Practice and Theory, 15(1), 32-46. https://doi. org/10.1016/j.simpat.2006.09.011.

Prof. Hyun-Woong Jin, Ph.D. Hannam University Department of Business Administration Korea jin@hannam.ac.kr 


\section{ANALYSIS OF FACTORS AFFECTING THE BENEFITS OF DEMAND INFORMATION SHARING}

\section{Hyun-Woong Jin}

Sharing customer demand information across the supply chain is known to be an effective approach to improve the performance of the whole supply chain. However, demand information sharing between companies requires a large amount of budget and leads to a change in the work process within the organization. Therefore, it is necessary to verify whether the sharing of customer demand information is beneficial to the company or not by considering their business environment. This paper aims to analyze the benefits of demand information sharing between companies in various business environments in order to provide managerial implications for the companies considering the adoption of a collaborative inventory management policy with external companies. This research uses a simulation approach based on system dynamics to model the considered supply chain and to explore its performance. For the simulation test, two types of simulation models were developed which represent a supply chain without information sharing and a supply chain with information sharing. Test results were analyzed in terms of the bullwhip effect, the inventory level and the stockout rate of the retailer. The results of this research may help practitioners to understand the dynamics of supply chain when the customer demand is shared. These understandings could help them to make a decision on adopting a collaborative inventory management policy based on the demand information sharing. The originality of this paper is that it deals with various business environments which are rarely considered in the previous researches. These include the length of ordering cycle, the maximum size of ordering quantity, backlog versus lost sales and the type of information shared.

Key Words: Information sharing, system dynamics, simulation, bullwhip effect, collaborative inventory management.

JEL Classification: M10, M11, C63.

DOI: 10.15240/tul/001/2019-3-013 